\title{
Cognitive Function Subordinate Domain
}

National Cancer Institute

\section{Source}

National Cancer Institute. Cognitive Function Subordinate Domain. NCI Thesaurus. Code C122365.

The collection of PROMIS item scales that assess perceived difficulties in intellectual processes, capabilities, and deficits. 